\title{
Efecto de estudiar biología celular con una célula virtual: comparación entre Canadá y Costa Rica, y entre educación presencial y a distancia
}

\author{
Víctor Hugo Méndez-Estrada y Julián Monge Nájera \\ Vicerrectoría de Investigación, Universidad Estatal a Distancia, 2050 San José, Costa Rica; vmendez@uned.ac.cr, julianmonge@gmail.com
}

Recibido 3-XI-2009 Corregido 15-XII-2009 Aceptado 7-II-2010

\begin{abstract}
Effect of studying cell biology with a virtual cell: a comparison between Canada and Costa Rica and between presential and distance education. When compared with education in industrialized countries, Latin American education often seems outdated and inferior. However, widespread use of computers might tend to reduce regional differences. To determine whether learning would be different when the same digital material was used in Canada (industrialized country) and Costa Rica (with an economy based on ecotourism and agriculture), we used Cellule, an interactive multimedia with animations, text and sound, for teaching basic cell biology. A total of 96 students from Canada and Costa Rica used the multimedia. We evaluated their opinion on the multimedia using questionnaires. We found no significant differences by country, or by the mode of teaching (in real classrooms or through distance learning). Cellule is an excellent multimedia for teaching basic cell biology, in both industrialized and Latin American countries.
\end{abstract}

\section{KEY WORDS}

Learning with computers, multimedia, virtual laboratories, digital cell, education in industrialized and non-industrialized countries.

\section{RESUMEN}

Cuando se le compara con la educación en los países industrializados, la educación latinoamericana suele parecer anticuada e inferior. Sin embargo, la computación generalizada podría tender a disminuir las diferencias regionales. Para averiguar si el aprendizaje sería diferente cuando un mismo material digital se usaba en Canadá (país industrializado) y Costa Rica (cuya economía se basa en el ecoturismo y la agricultura), usamos Cellule, un material multimedial interactivo con animaciones, texto y locución, para la enseñanza de la biología celular básica. Participaron en total 96 estudiantes y evaluamos su opinión mediante cuestionarios. No encontramos diferencias importantes según el país, o la modalidad de enseñanza (presencial o a distancia). El curso virtual Cellule es un excelente material para la enseñanza de la biología celular básica, tanto en países industrializados como en países latinoamericanos.

\section{PALABRAS CLAVE}

Aprendizaje con computadoras, multimedia, laboratorio virtual, célula digital, subdesarrollo.
Cuando se le compara con la educación en los países industrializados, la educación latinoamericana suele parecer anticuada e inferior en todos los aspectos. Se enseñan conocimientos automatizados y repetitivos que poco fomentan el desarrollo de la iniciativa, la creatividad o la capacidad para comunicarse a través de distintas medios (Cuicas 2006).

Nuestra impresión tras asistir por más de una década a diversos congresos internacionales es que los educadores latinoamericanos se pierden en inacabables divagaciones filosóficas sobre el "modelo educativo" y "cómo podrían aplicarse las nuevas tecnologías", mientras que los colegas de países industrializados presentan casos exitosos de experiencias innovadoras y a veces, sorprendentemente sencillas y eficaces.

Por supuesto, el uso de la computadora no es la solución para todos los problemas educativos, pues lo básico es aprender a construir significados y dar sentido a lo que se aprende (Ausubel et al. 1983, Coll et al. 1992). Pero el uso de las tecnologías de la información y comunicación (TIC) ayuda a acercar a docentes y estudiantes y permite difundir hallazgos mediante sitios como Wikipedia, que a inicios del 2010 tenía cerca de 14000000 de artículos en 262 idiomas (wikipedia.com) y YouTube, donde hay pequeños videos que enseñan a hacer casi cualquier actividad (youtube.com), desde el ámbito artístico 
(por ejemplo, como tocar la flauta dulce) hasta el técnico (por ejemplo, cómo editar una fotografía digital).

Las tecnologías de la información y comunicación (TIC) ofrecen estrategias metodológicas que brindan varias ventajas pedagógicas: incorporación al sistema de un mayor número de personas que tienen un papel más activo, responsable y autónomo (Sangrà \& Duart 2000, Herrera 2005, Area 2006). Además, mediante el uso de wikis (documentos digitales construidos comunalmente), blogs (sitios web que se actualizan periódicamente), foros, videoconferencias y pláticas (chats), permiten captar la complejidad de la realidad de los procesos de enseñanza y aprendizaje, tanto en las modalidades presenciales como a distancia (Aular 2000, Monge-Nájera et al. 2001, Carballo 2006, Cuicas 2006).

La computadora permite aprender al propio ritmo, sin necesidad de asistir a lecciones en horarios y sitios definidos. Permite desvelar lo oculto a los sentidos, así como apoyarlo con movimientos y sonidos, por ejemplo para estudiar procesos y comportamientos de una forma muy superior a los diagramas de los viejos textos impresos (García 2000, Candelas et al. 2004, Alejandro 2004, Herrera 2005, Carballo 2006, Casadei 2006).

Sin embargo, no es lo mismo pretender usar computadoras para educar en un país industrializado, donde buena parte de la población tiene acceso a ellas y conexión a Internet, que en un país latinoamericano. Quisimos por eso hacer una comparación entre dos países contrastantes en población, economía y educación: Costa Rica, en América Central, y Canadá. Elegimos para ello el tema de la célula, ya que este es un tema biológico que se estudia en todo el mundo, lo que daría mayor aplicabilidad a nuestros resultados. Usamos para el estudio un material multimedial interactivo con animaciones, texto y locución. Finalmente, quisimos comparar el efecto de estudiar presencialmente con docente, y a distancia, por lo que tuvimos dentro de Costa Rica un grupo adicional que usó el material a distancia, sin docente presencial. Este informe compara la percepción que tuvo el estudiantado según su pertenencia a esos tres grupos: país industrializado; país centroamericano, y presencial versus a distancia.

\section{METODOLOGÍA}

Usamos para el estudio Cellule, un material multimedial interactivo con animaciones, texto y locución elaborado por Jean-Marc Grandbois, de la Universidad de Quebec, Canadá. Contiene un ambiente de aprendizaje dinámico y amigable, relacionado con conceptos básicos de la célula, en un disco compacto autoejecutable, que funciona sin conexión de Internet. Según el país, se usaron versiones en francés y español. El material propone preguntas de evaluación formativa para comprobar el propio avance.

Fue evaluado en cuatro grupos de estudiantes y con dos modalidades de enseñanza distintas: a distancia con 31 estudiantes (32\% de la población) de Biología I del Profesorado en la Enseñanza de las Ciencias de la Universidad Estatal a Distancia, Costa Rica; presencial (68\%), con 20 estudiantes (21\%) de Biología y Genética de la carrera de Medicina y Cirugía de la Universidad de Iberoamérica, Costa Rica; con 29 estudiantes (30\%) de Histofisiología animal y con 16 (17\%) de Fisiología humana de la carrera de Enfermería de la Université du Québec à Rimouski, Canadá, para un total de 96 estudiantes.

Los datos fueron procesados y analizados en el Programa Estadístico para Ciencias Sociales (SPSS) 15.0 v. Se realizaron pruebas de Chi cuadrado y de Fisher.

\section{RESULTADOS}

\section{Resultados en que no hubo diferencia entre grupos}

La mayoría de las personas participantes en el estudio son mujeres menores de 23 años (Cuadro 1). Calificaron su experiencia en computación como regular (66\%), mucha (20\%) o poca (14\%).

En general usan con frecuencia un procesador de texto, Internet y el correo electrónico; pero desconocen o usan muy poco los programas PowerPoint, Excel, Dreamweaver, Photoshop y Access.

Normalmente usaron el laboratorio virtual en la casa (58\%); pocas donde un amigo, en el lugar de trabajo, café internet u otro. El 68\% leyeron las instrucciones antes de utilizar el laboratorio; el $90 \%$ las consideraron claras y el $78 \%$ no tuvieron problemas en el disco.

La apariencia fue considerada excelente (37\%), buena $(48 \%)$ o regular (15\%). En ambos grupos, menos del $30 \%$ opinó que los íconos siempre fueron suficientes y claros, y la presentación siempre fue dinámica, de fácil avance y agradable.

La mayoría (80\%) no encontró dificultad para realizar las actividades. El $20 \%$ que las tuvo indicó como causas la falta de claridad metodológica, las actividades poco específicas, el no entender, las imágenes estáticas, el no ver simultáneamente texto e imagen y la falta de conocimientos en computación.

El 70\% logró los objetivos de aprendizaje (26\% en forma parcial). El 30\% que no lo logró mencionó como causas 


\section{CUADRO 1}

Sexo, según el curso y la modalidad de enseñanza de los estudiantes que hicieron el laboratorio virtual de la célula (total 96 casos)

\begin{tabular}{|c|c|c|c|c|c|}
\hline \multirow{2}{*}{ Curso } & \multicolumn{2}{|c|}{ Presencial } & \multicolumn{2}{|c|}{ A distancia } & \multirow{2}{*}{ Total (\%) } \\
\hline & $\%$ Masculino & $\%$ Femenino & $\%$ Masculino & $\%$ Femenino & \\
\hline Biología I ( $\mathrm{n=31)}$ & - & - & 15 & 18 & 33 \\
\hline Histofisiología animal $(n=29)$ & 9 & 21 & - & - & 30 \\
\hline Biología y genética $(n=20)$ & 9 & 11 & - & - & 20 \\
\hline Fisiología humana $(n=16)$ & 2 & 15 & - & - & 17 \\
\hline Total $(n=96)$ & 20 & 47 & 15 & 18 & 100 \\
\hline
\end{tabular}

que los contenidos fueron tratados superficialmente, no contar con toda la información y bibliografía extra, la falta de ilustraciones dinámicas, lo cansado de estudiar en computadora, los temas tan especializados y la ausencia del docente. Solo un 30\% indicó que las actividades demandan un tiempo excesivo.

Las recomendaciones generales fueron aumentar la interactividad, reducir el texto, incluir glosario y respuestas a las preguntas de autoevaluación y que el laboratorio sea autosuficiente.

\section{Resultados en que difirieron el grupo presencial y el grupo a distancia}

Los problemas mencionados por quienes tomaron el curso presencial fueron que el sonido no funcionaba, que la locución se repetía en lugares incorrectos, que algunas actividades no funcionaban, que el disco no abría, que no se veían los dibujos y que había frases en francés en la parte en español. A distancia tuvieron los mismos problemas para abrir el laboratorio, ver los dibujos y la locución francesa desubicada; pero además no lograron usar algunas partes o imprimir el material.

En el grupo presencial, más del 30\% consideró que siempre fueron buenas la estructura del material digital y la relación entre el texto y las ilustraciones, así como la apariencia y cantidad de las ilustraciones. Hubo menor satisfacción para estas características en el grupo a distancia (menos del 16\%).

El grupo a distancia halló más difícil responder las preguntas ( 20 de 30 estudiantes) que el presencial ( 15 de 65; Prueba Exacta de Fisher; $P=0,00008$ ). La causa principal fue que el texto no incluye suficiente información para responder las preguntas (56\%), no se suministra bibliografía adicional (20\%), algunas preguntas no estaban bien planteadas (18\%), la ventana que se abre es muy grande y no permite ver los botones (4\%), y que algunas preguntas no tienen relación con el contenido (2\%).

\section{DISCUSIÓN}

La juventud de quienes tomaron el curso se explica porque el tema de la célula se incluye en un curso básico para estudiantes de primer ingreso al sistema universitario.

Un estudio previo en Venezuela halló oposición estudiantil a la posibilidad de cambiar un curso a la modalidad en línea porque su complejidad requería el asesoramiento e interacción permanente del personal docente (Aular 2006), Sin embargo, actualmente los entornos virtuales rompen las barreras de tiempo y espacio geográfico y permiten aprender los mismos contenidos en diversas latitudes (Didier 1998, Aular 2006, Carballo 2006, Cuicas 2006, Salas 2006). En nuestro caso, hallamos similares niveles de conocimiento práctico de la computación en Canadá y Costa Rica. La evaluación estudiantil pidió más actividades virtuales interactivas y calificaron la metodología como excelente para aprender sobre el complejo tema de la estructura, funcionamiento y fisiología de la célula. El que una tercera parte de los estudiantes de la modalidad a distancia considere que el material es insuficiente para responder las preguntas, es comprensible si tenemos que los estudiantes de la UNED esperan que los materiales didácticos den toda la información necesaria para no tener que consultar otras fuentes. Los resultados de Ortega et al. (2006) indican que los estudiantes analizan como favorablemente la idea de tener un curso virtual que se pueda consultar a distancia, pero casi unánimemente consideran necesaria la presencia de un docente que "imparta las clases, sobre todo porque piensan que es más simple acudir al profesor 
en persona en caso de que surja alguna duda que aclarar la misma por medio de la computadora." (pág. 11).

Un estudio encontró relación entre el uso de estrategias basadas en el software matemático y el aprendizaje (Cuicas 2006). Por otros estudios, nosotros creemos que el valor del uso las computadoras en la enseñanza está en función de lo que se diseña, planea y evalúa, independientemente del país o la modalidad (Monge-Nájera et al. 2003, 2007, Méndez \& Monge-Nájera 2004, 2006, Méndez \& Delgado 2006). En nuestro estudio, el que no existiera asociación importante entre el aprendizaje según el país, o la modalidad de enseñanza (presencial o a distancia), calza con esta idea y corrobora que el curso virtual Cellule, creado y elaborado por Jean-Marc Grandbois, en la Universidad de Quebec, es un excelente material para la enseñanza de la biología celular básica, tanto en países industrializados como en países latinoamericanos.

\section{AGRADECIMIENTOS}

Agradecemos especialmente a Jean-Marc Grandbois por permitirnos traducir al español y utilizar Cellule, así como por autorizar amablemente el uso de los datos canadienses en esta publicación. También al estudiantado participante en la Universidad de Quebec (campus de Rimouski), la Universidad Estatal a Distancia y la Universidad de Iberoamérica (ambas en San José, Costa Rica).

\section{REFERENCIAS}

Alejandro, C. A. 2004. Prácticas de laboratorio de Física general en Internet. Departamento de Física. Universidad Central "Marta Abreu" de las Villas. Cuba. Revista Electrónica de Enseñanza de las Ciencias 3. (http://www.saum.uvigo. es/reec/volumenes/volumen3/Numero2/ART6_Vol3_ N2.pdf. ; consultado el 24 de julio)

Area, M. 2000. ¿Qué aporta Internet al cambio pedagógico en la educación superior? Publicado en Redes multimedia y diseños virtuales. Actas del III Congreso Internacional de Comunicación, Tecnología y Educación. Universidad de Oviedo, 12-15 setiembre, Oviedo, España.

Aular, A. 2006. Necesidades instruccionales: Oportunidades de intervención tecnológica. XIII Congreso Internacional de Tecnología y Educación a Distancia

Ausubel, D.P., Novak, J.D. y Hanesian, H. 1983. Psicología Educativa. Trillas, Distrito Federal, México.

Candelas, F., F. Torres, P. Gil y F. Ortiz. 2004. Laboratorio virtual remoto para robótica y evaluación de su impacto en la docencia. Santiago Puente, Jorge Pomares Dpto. de Física, Ingeniería de Sistemas y Teoría de la Señal Escuela Politécnica Superior, Universidad de Alicante. Alicante,
España. (http://riai.isa.upv.es/CGI-BIN/articulos\%20revisados\%202004/num2/414/riai-2004414impr.pdf.; consultado el 24 de julio, 2006)

Carballo, K. 2006. Tecnología educativa para la enseñanza de la geografía. XIII Congreso Internacional de Tecnología y Educación a Distancia, Costa Rica UNED, 1- 3 de noviembre , San José, Costa Rica.

Casadei, L. El uso de simulaciones como herramienta de apoyo para la comprensión de conceptos en física. XIII Congreso Internacional de Tecnología y Educación a Distancia, Costa Rica UNED, 1 - 3 de noviembre , San José, Costa Rica.

Coll, C., Pozo, J., Sarabia, B., y Valls, E. 1992. Los contenidos de la reforma: Enseñanza y aprendizaje de conceptos, procedimientos y actividades. Santillana, Madrid, España.

Cuicas, M. 2006. El software matemático como herramienta para el desarrollo de habilidades del pensamiento. XIII Congreso Internacional de Tecnología y Educación a Distancia, Costa Rica UNED, 1-3 de noviembre, San José, Costa Rica.

Didier, O. 1998. Debate temático: de lo tradicional a lo virtual: las nuevas tecnologías de la información. Fondo Francés de Información, París.

Sangrà, A. \& J. Duart. 2000. Aprender en la virtualidad. Gedisa, Catalunya, España.

García. J. L. 2000. Entornos virtuales de enseñanza. ¿Un sistema didáctico? Contexto Educativo. Revista Digital de Educación y Nuevas Tecnologías. Núm. 28, Año V. (http://contexto-educativo.com.ar/2003/4/nota-06.htm.; consultado 5 de setiembre, 2006)

Herrera, L. 2005. Las tecnologías de la información y las comunicaciones en la enseñanza de la Historia. Contexto Educativo. Revista Digital de Educación y Nuevas Tecnologías. Año VI (36). (http://contexto-educativo.com.ar/2005/3/ nota-07.htm..; consultado 5 de setiembre, 2006)

Méndez-Estrada, V. H. \& E. Delgado. 2006. La revisión de la práctica educativa para el rediseño del curso de Metodología de la enseñanza de las Ciencias Naturales. XIII Congreso Internacional sobre Tecnología y Educación a Distancia. La educación a distancia ante los desafíos del conocimiento, 3-5 de noviembre, San José, Costa Rica.

Méndez-Estrada, V. H. 2006. Currículo de un curso de Zoología basado en un libro de texto descriptivo a un currículo sustentado en multimedios. XIII Congreso Internacional sobre Tecnología y Educación a Distancia. La educación a distancia ante los desafíos del conocimiento, 3-5 de noviembre, San José, Costa Rica.

Méndez-Estrada, V. H. y J. Monge-Nájera. 2004. Enseñanza a distancia de la biología general mediante un libro de texto: enseñanzas de una experiencia. Universidad Estatal a Distancia. XII Congreso Internacional sobre Tecnología y Educación a Distancia, , 3 al 5 de noviembre, San José, Costa Rica.

Méndez, V. H. \& J. Monge-Nájera. 2006. Opinión de los estudiantes acerca del uso de las tecnologías de la información y 
comunicación empleadas como parte de la evaluación en el curso de Biología General, II cuatrimestre, 2005. Centro de Mejoramiento de los Procesos Académicos (CEMPA), Universidad Estatal a Distancia, San José, Costa Rica

Monge-Nájera, J.A., M. Rivas Rossi \& V.H. Méndez-Estrada. 2001. Internet, Multimedia and Virtual Laboratories in a "Third World" Environment. Open Learning 16: 279-290.

Monge-Nájera, J; M. Rivas y V. H. Méndez-Estrada. 2003. Enseñanza a distancia de estrategia empresarial: opinión de estudiantes y docente sobre el uso de material multimedial y recomendaciones para mejorar su eficacia. X Encuentro Iberoamericano de Educación Superior a Distancia, 21 23 de julio, San José, Costa Rica.
Monge Nájera J. y V. H. Méndez Estrada. 2007. Ventajas y desventajas de usar laboratorios virtuales en educación a distancia: la opinión del estudiantado en un proyecto de seis años de duración. Universidad de Costa Rica. Revista de Educción 31:91-108.

Ortega, J., M. Martínez y Á. Rodríguez. 2006. Educación a distancia: experiencias en la impartición de un curso de Física II para estudiantes de la carrera de Ingeniería Informática. XIII Congreso Internacional de Tecnología y Educación a Distancia UNED, 1-3 de noviembre, San José, Costa Rica.

Salas, I. 2006. Entornos virtuales: nuevos escenarios para el aprendizaje. XIII Congreso Internacional de Tecnología y Educación a Distancia UNED, 1-3 de noviembre, San José, Costa Rica. 
